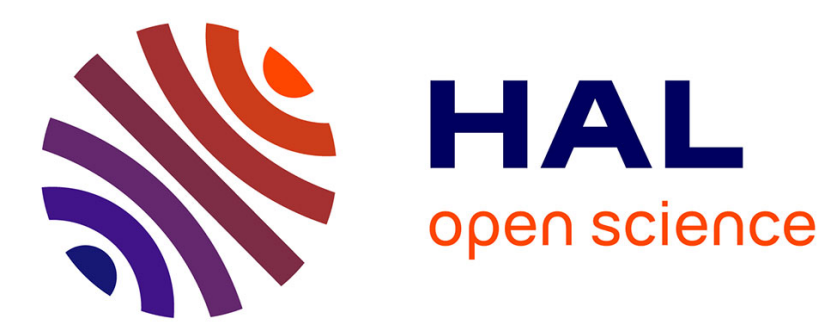

\title{
Interdépendances et conflictualités russo-européennes en matière de gaz naturel \\ Catherine Locatelli
}

\section{To cite this version:}

Catherine Locatelli. Interdépendances et conflictualités russo-européennes en matière de gaz naturel. La revue internationale et stratégique, 2011, 84, pp.95-104. halshs-00653328

\section{HAL Id: halshs-00653328 \\ https://shs.hal.science/halshs-00653328}

Submitted on 19 Dec 2011

HAL is a multi-disciplinary open access archive for the deposit and dissemination of scientific research documents, whether they are published or not. The documents may come from teaching and research institutions in France or abroad, or from public or private research centers.
L'archive ouverte pluridisciplinaire HAL, est destinée au dépôt et à la diffusion de documents scientifiques de niveau recherche, publiés ou non, émanant des établissements d'enseignement et de recherche français ou étrangers, des laboratoires publics ou privés. 


\section{ÉCONOMIE DU DÉVELOPPEMENT DURABLE ET DE L'ÉNERGIE}

\section{Interdépendances et conflictualités russo-européennes en matière de gaz naturel}

Catherine Locatelli

septembre 2011

note de travail $n^{\circ} 4 / 2011$ 



\section{Interdépendances et conflictualités russo-européennes en matière de gaz naturel Catherine L ocatelli, chargée de recherche CNRS \\ EDDEN-LEPII, U niversité de Grenoble, CNRS \\ BP $47, \mathrm{~F}-38040$ Grenoble cedex 9 \\ Email : catherine.locatelli@upmf-grenoble.fr \\ Tel : 33456528575}

Septembre 2011

La Russie dont la compagnie Gazprom détient le monopole d'exportation de gaz naturel, est un fournisseur majeur de l'UE. De la même manière, l'Europe est le marché d'exportation privilégié de la Russie. En dépit de ces intérêts convergents, les relations entre les deux partenaires s'avèrent depuis le début des années 2000 extrêmement conflictuelles, largement dominées par des rapports de défiance mutuelle. En dépit des interdépendances économiques, les questions de sécurité énergétique sont au cœur du partenariat énergétique qui se négocie entre les deux zones. L'Etat russe contrôlant Gazprom est accusé d'utiliser «l'arme gazière » dans ses relations avec I'UE. Celle-ci est par ailleurs supposée de par sa politique énergétique et climatique faire peser un risque spécifique sur les producteurs gaziers.

Les difficultés de la relation gazière UE - Russie expriment d'abord une vision différenciée des règles et régulations devant présider à la structuration des marchés et à l'organisation des industries gazières. A la vision concurrentielle et multilatérale de I'UE s'oppose celle de la Russie centrée sur les compagnies d'Etat et privilégiant des relations bilatérales. Ces deux visions sont à l'évidence le produit de deux environnements institutionnels très différenciés qui ont conduit l'Etat russe à mettre profondément en cause le pouvoir normatif de I'UE. M ais les difficultés rencontrées sont aussi l'expression de l'incapacité de I'UE à définir et exprimer une politique commune par rapport à la R ussie. Dès lors, les relations entre les deux zones se structurent essentiellement au niveau des compagnies gazières et des Etats membres.

\section{L es interdépendances gazières UE -R ussie}

Les relations gazières entre I'UE et la Russie sont marquées par des relations d'interdépendance et non par une simple dépendance d'une zone par rapport à une autre. L'UE est dépendante du gaz russe pour son approvisionnement, mais à des degrés divers selon les pays considérés. Elle peut par ailleurs recourir à d'autres fournisseurs gaziers, notamment avec le dével oppement du GN L ce qui concoure à sa sécurité gazière. A l'inverse, la Russie témoigne d'une extrême dépendance par rapport à l'Europe en matière de marchés d'exportation. Si des stratégies de diversification sont à ce jour envisagées par Gazprom et l'Etat russe, elles sont pour le moment loin de leur concrétisation et rencontrent toujours sur un certain nombre de contraintes.

\section{$1.1 \mathrm{~L}$ a dépendance européenne, des situations contrastées}

Les exportations de gaz naturel de Gazprom vers l'Europe sont importantes. Elles se sont chiffrées en 2009 à $156 \mathrm{Gm}^{3}$. Toutefois, les situations sont très variables selon les pays. Trois grands importateurs émergent en termes de volume: I'Allemagne, la France et I'Italie. L'Allemagne importe $33,5 \mathrm{Gm}^{3}$ de gaz en provenance de Russie. Elle est surtout moins diversifiée en termes de fournisseurs que la France et I'Italie, la Russie représentant près de $40 \%$ de ses importations. De ce point de vue, la situation des pays Baltes et des pays d'Europe centrale est à souligner. Si les volumes importés sont faibles, les dépendances sont 
extrêmes (cf. tableau 1). Des raisons historiques (CAEM) mais aussi géographiques expliquent une telle situation. A l'inverse, I'Espagne, la B el gique voire le R oyaume-Uni, sont peu impliqués dans les échanges gaziers avec la Russie. On peut donc s'attendre au sein de I'Union à des points de vue différenciés quant à la question du « risque russe ». La sécurité d'approvisionnement en gaz naturel provenant de Russie est ainsi I'élément central du débat européen relatif aux enjeux de sécurité énergétique.

\section{Tableau 1 : Dépendance de quelques pays de l'UE vis-à-vis du gaz russe}

\begin{tabular}{|c|c|c|}
\hline Pays & Volumes importés, $\mathbf{G ~ m}^{3}$ & $\begin{array}{l}\text { Part de marché de Gazprom } \\
\text { (/importations de Gaz N), \% }\end{array}$ \\
\hline Allemagne & 33,5 & 35,5 \\
\hline A utriche & 5,4 & 68,2 \\
\hline B elgique & 3,3 & 15,3 \\
\hline Bulgarie & 2,2 & 100,0 \\
\hline Estonie & 0,8 & 100,0 \\
\hline Finlande & 4,4 & 100,0 \\
\hline France & 10,0 & 16,7 \\
\hline Grèce & 2,1 & 62,3 \\
\hline Italie & 19,1 & 30,0 \\
\hline Lettonie & 1,1 & 100,0 \\
\hline Lituanie & 2,5 & 100,0 \\
\hline Hongrie & 7,6 & 88,9 \\
\hline Pologne & 9,0 & 78,1 \\
\hline Rep. tchèque & 7,1 & 68,1 \\
\hline Roumanie & 2,5 & 100,0 \\
\hline Royaume-Uni & 9,7 & 26,6 \\
\hline
\end{tabular}

Sources : Gazprom, Rapport 2010, M oscou ; B P Energy statistical review, 2010

\subsection{L'UE, le « marché rentable » de la R ussie}

La dépendance économique de Gazprom et de la Russie par rapport à l'Europe est considérable. Celle-ci est un marché essentiel pour Gazprom puisqu'elle représente près de $71 \%$ de ses exportations à coté de celui de la CEI. Les exportations de GNL vers I'A sie (notamment le Japon) à partir de Sakhaline restent marginales même si elles sont en augmentation. L'Europe est surtout le marché rentable pour Gazprom, en comparaison de celui de la CEI mais aussi de son marché intérieur, en raison de différences de prix sur les trois marchés. Ces disparités de prix restent considérables en dépit des augmentations enregistrées depuis la fin des années 2000 sur le marché intérieur russe ou sur les marchés de la CEI. Des logiques de formation des prix différenciées en sont I'explication ${ }^{1}$. En 2010, le prix moyen d'exportation a été de $310 \$ / 1000 \mathrm{~m}^{3}$ vers l'E urope contre en moyenne $63 \$ / 1000$ $\mathrm{m}^{3}$ pour les ménages russes et $82 \$ / 1000 \mathrm{~m}^{3}$ pour l'industrie.

Gazprom est d'autant plus dépendant du marché européen qu'à ce jour la compagnie ne dispose pas de réelles voies de diversification, en dépit de sa stratégie d'exportation vers I'A sie ou les Etats-Unis au travers de la filière GNL. Les évolutions du marché gazier

\footnotetext{
${ }^{1}$ Les prix des exportations vers la CEI ont longtemps été basés sur une approche cost-plus. Ces exportations sont donc beaucoup moins rentables que celles à destination de I'UE dominées par les logiques et les principes du netback au sein des contrats Take or Pay (K onoplyanik, 2010).
} 
américain, suite à la mise en production des shale gas, laissent douter au moins sur le court terme de la rentabilité d'exportations de GNL à partir de la Russie. Quant à I'Asie, une politique d'exportation conséquente n'est envisageable que sur le long terme. De nombreuses contraintes, développement des infrastructures et des gisements, restent à surmonter. Par ailleurs, il n' est pas sûr que le marché chinois assure à Gazprom le même niveau de rentabilité que le marché européen pour ses exportations. La signature d'un contrat gazier de $30 \mathrm{Gm}^{3}$ par an entre la Russie et la Chine, le projet A Itaï, bute toujours sur la formule d'indexation des prix. A court terme, les Chinois semblent considérer que le gazoduc turkmène suffit à satisfaire leur demande en gaz naturel ${ }^{2}$.

\section{Une vision différenciée des marchés et des industries du gaz naturel}

En dépit de ce contexte d'interdépendances économiques fortes, le partenariat gazier entre I'UE et la Russie se caractérise paradoxalement par des incompréhensions persistantes qui témoignent de difficultés à dialoguer. D'un côté, la Russie est apparue de plus en plus aux yeux de l'Europe comme posant un risque spécifique quant à sa sécurité d'approvisionnement. De leur côté, les russes considèrent que leur stratégie d'exportation est mise en cause par les évolutions du marché gazier européen. Face à l'incapacité de l'UE à définir une politique et une position commune concernant la Russie, les relations bilatérales prédominent que ce soit entre chaque pays de I'UE et la Russie, ou entre les compagnies gazières, et se focalisent sur les seuls enjeux économiques.

\subsection{Les oppositions économiques de court terme}

Les questions de sécurité, dans ses deux aspects offre et demande, focalisent aujourd'hui un certain nombre d'oppositions économiques entre la Russie et I'UE. L'UE considère que sa sécurité d'approvisionnement (sécurité de l'offre) est mise en cause par la stratégie d'investissements de Gazprom. Cette dernière serait l'expression du pouvoir de marché que la compagnie gazière tenterait d'exercer sur son principal marché d'exportation. La Russie, quant à elle, redoute les incertitudes de la demande gazière de I'UE (risque demande) créées par la politique de libéralisation des industries de réseau et la politique climatique de I'Europe.

- Les enjeux de la libéralisation des marchés gaziers de l'U E : la sécurité de la demande

La libéralisation du marché gazier européen déstabilise les relations contractuelles avec les fournisseurs traditionnels de I'UE. En modifiant ou en supprimant certaines clauses des contrats de long terme Take or Pay, (formule d'indexation des prix, clause de destination de finale, clause relative à la flexibilité des enlèvements), elle accroît le « risque prix » et le « risque volume» pour les producteurs gaziers (Boussena, 1999). Le troisième paquet énergie est également porteur d'enjeux importants pour les fournisseurs de I'UE. Certaines règles pourraient sensiblement limiter les investissements de Gazprom en Europe, mettant ainsi en cause la stratégie industrielle développée par la société. L'introduction d'un unbundling patrimonial ${ }^{3}$, dans le cadre de la dé-intégration de son industrie gazière, condamnerait toute

\footnotetext{
${ }^{2}$ Le central A sia-China gas pipeline permet au Turkménistan d'exporter $40 \mathrm{Gm}^{3}$ de gaz naturel vers la Chine. A ce jour, la CNPC a signé un contrat de $30 \mathrm{Gm}^{3}$ de gaz à partir de 2012. (Turkmenistan puts its foot on the gas, Petroleum Economist, Oct. 2010, p. 16).

${ }^{3}$ L'action d'unbundling consiste en une séparation juridique ou comptable des divers segments de la chaîne gazière pour éviter l'intégration verticale considérée comme une barrière à l'entrée pour les nouveaux acteurs.
} 
stratégie de descente en aval et d'intégration verticale sur le marché européen. Le compromis final est moins ambitieux que les propositions initiales de la Commission européenne ${ }^{4}$. M ais les règles adoptées excluent qu'un producteur et fournisseur comme la Russie puisse être en même temps un gestionnaire de réseau (Transmission System Operator ou TSO) dans un Etat membre (W illems, Sul, B enizri, 2010). Enfin, la «third country clause » ouvre la voie à un traitement discriminatoire des investissements étrangers. Sel on cette dernière, les compagnies détenues par des actionnaires hors UE devront démontrer qu' elles ne portent pas atteinte à la sécurité énergétique de l'UE si elles veulent pouvoir opérer dans I'U nion.

\section{- La stratégie d'investissements de Gazprom en question : la sécurité de l'offre}

La sécurisation d' une offre gazière suffisante et à un coût acceptable est aujourd' hui un enjeu majeur pour I'UE. De ce point de vue, la R ussie a pu constituer un risque particulier au moins concernant trois facteurs. En premier lieu, I'UE a pendant un certain temps estimé que les investissements de Gazprom dans le développement de nouveaux gisements étaient insuffisants pour compenser le déclin des trois "supers géants », U rengoi, Y amburg et Medvezhe (M ilov, 2005 ; Riley, 2006 ; Stern, 2006). Ceci a pu faire douter de la capacité de la société gazière à faire face à moyen terme à ses engagements contractuels à l'export. Les évolutions récentes du marché gazier européen, baisse de la demande avec la crise économique actuelle et développement des shale gas, ont allégé cette contrainte mais n'enlèvent pas toute incertitude sur le devenir de la production de Gazprom. En effet, face à son surplus de production actuel la compagnie maintient son attentisme en matière de développement de nouveaux gisements à l'image de Shotkman (B oussena, L ocatelli, 2011).

Cette incertitude sur l'offre gazière russe est parfois considérée comme l'expression de la volonté de Gazprom d'exercer un éventuel pouvoir de marché sur le marché gazier de l'UE. Celui-ci consisterait en une stratégie visant notamment à réduire les volumes offerts (en particulier sur les marchés spot) afin d'augmenter les prix. M ême si elle reste très limitée, la politique de descente vers l'aval des marchés européens menée par Gazprom depuis le milieu des années 1980 en réponse à la libéralisation des marchés gaziers de l'UE s'inscrirait également dans une telle logique. La création d'une OPEP du gaz, en s'appuyant sur le souhait de certains autres producteurs de développer une politique coordonnée des productions de gaz naturel afin de limiter les quantités produites et soutenir les prix, serait l'expression la plus achevée de l' exercice de ce pouvoir de marché.

Le deuxième facteur d'incertitude pour l'UE réside dans les évolutions législatives russes. Celles-ci rendent l'accès aux ressources en hydrocarbures de la Russie de plus en plus contraignant et limité pour les compagnies pétrolières et gazières internationales (cf. encadré 1). Or, dans le contexte de libéralisation de ses marchés gaziers, les investissements des compagnies européennes dans les pays producteurs d'énergie et dans les pays de transit sont un élément essentiel de la sécurité gazière européenne (European Commission, 2010).

La sécurité du transit est le dernier facteur perçu comme menaçant la sécurité d'approvisionnement de I'UE. Les différentes crises gazières de 2006 et 2008 entre I'U kraine et la R ussie (voire celle avec la B iélorussie en matière pétrolière), ont porté au grand jour les difficultés de ces pays à normaliser leurs relations économiques et à mettre fin au système de prix hérité de l'économie planifiée.

\footnotetext{
${ }^{4}$ Chaque pays peut choisir entre adopter un unbundling patrimonial ou un schéma dans lequel est instauré un transmission system operator (TSOs).
} 


\section{E ncadré 1 : L'accès aux hydrocarbures russes, les principales dispositions}

Différents amendements ont été introduits dans la loi sur les ressources en terre (Subsoil resources Law) signée en 1992 :

- Le principe d'allocation conjointe par l'Etat et les régions (two-key principle) pour les licences d'exploration et de production a été annulé ( $L$ eB oeuf, $L a m b, G$ reene and $M$ acR ae, 2005). Seul I'E tat peut désormais attribuer de telles licences.

- Une liste de gisements stratégiques a été définie, pour lesquels les licences d'exploration-production ne peuvent être attribuées qu' aux seules compagnies pétrolières d'E tat russes

- Seules les compagnies d'Etat russes (c'est-à-dire Rosneft et Gazprom) peuvent développer les ressources en hydrocarbures offshores.

L a loi sur les investissements étrangers dans les secteurs stratégiques promulguée en 2008 s'applique au secteur des hydrocarbures. Elle limite les investissements étrangers à $10 \%$. A u-delà, une autorisation spéciale est nécessaire.

\subsection{Des visions divergentes sur la structure des marchés et des industries gazières}

Ces risques économiques sont l'expression de conflits d'intérêts de la part des acteurs économiques en présence. $\mathrm{M}$ ais au-delà, ils reflètent des conceptions contradictoires quant à la structuration des marchés et des industries gazières en fonction d'environnements institutionnels différents. Dans le contexte nouveau de libéralisation de ses marchés gaziers, I'UE entend gérer les relations avec ses fournisseurs de gaz naturel au travers de la diffusion de ses acquis communautaires en matière de concurrence et de réglementation des marchés énergétiques (McGowan, 2007). L'objectif est à terme d'établir un espace commun de régulation, de normes et de règles (Belyi, 2009). Cela suppose des modèles d'organisation calqués sur ceux définis par les différentes directives électriques et gazières européennes, à savoir un modèle d'organisation dé-intégré des industries de réseau. La diffusion des acquis communautaires aux pays producteurs permettrait en particulier à l'UE de sécuriser son offre gazière, en garantissant la possibilité pour ses compagnies gazières d'accéder aux ressources en hydrocarbures des producteurs. Cela constitue sans doute un objectif stratégique pour I'UE.

Toutefois, les modèles d'organisation des marchés et des industries promus par l'UE se trouvent en totale contradiction avec la politique énergétique et économique définie par la Russie. Cette dernière entend mettre le gaz et le pétrole au service de sa puissance et de sa croissance économique. Cela impose à l'Etat de définir des modèles d'organisation et de régulation des industries d'hydrocarbures qui lui permettent de répondre à ces deux principaux objectifs. Dans le contexte institutionnel russe, il ne peut guère $s$ appuyer sur les outils traditionnels mis en œuvre dans les économies de marché développées (exemple de la Norvège), notamment une fiscalité, des contrats pétroliers et des régulations extrêmement détaillés. Dès lors, le recours aux compagnies d'E tat et le contrôle plus strict, par les autorités fédérales, des conditions d'accès aux ressources en hydrocarbures, sont les deux voies utilisées pour tenter de pallier l'ineffectivité des institutions de marché telles que définies dans les économies dével oppées. A l'évidence, les modalités et les principes de cette réorganisation ne suivent pas la logique de l'approche concurrentielle défendue par l'UE. 
La Russie et l'UE sont condamnées, au moins sur le court terme, à s'entendre en dépit des divergences actuelles, tant sont importantes les interdépendances gazières entre les deux zones. A plus ou moins long terme, les scénarios peuvent être plus ouverts. La Russie pourrait disposer d'autres zones potentielles d'exportation centrées sur le marché asiatique et I'UE d'autres fournisseurs gaziers comme les producteurs de la Caspienne et du Moyen-Orient. C'est à l'évidence l'un des enjeux du pipeline Nabucco.

\section{Références}

B elyi, A. (2009) Reciprocity as a factor of the energy investment regimes in the EU-Russia energy relations. J ournal of World Energy Law \& Business 2(2) 117-127.

Boon von Ochssée, T. (2010) The dynamics of natural gas supply coordination in a new world. Cligendael International Energy Programme, CIEP, $429 \mathrm{p}$.

B oussena. S. and L ocatelli. C. (2011) Gas market developments and their effect on relations between R ussia and the EU. OPEC Energy Review 35(1) 27-46.

B oussena, S. (1999) New European Gas M arket: Gas Strategies of Other Present and Potential Suppliers, The 1999 International Conference: The role of Russian and CIS Countries in Deregulated Energy Markets (The M oscow International Energy Club et le Centre de Géopolitique des Matières Premières-U niversité Paris Dauphine), Paris, 6-7 décembre.

European Commission (2010) Energie 2020: Stratégie pour une énergie compétitive, durable et sûre. Communication de la Commission au Parlement Européen, au conseil, au comité économique et social européen et au comité des régions, COM 639.

Konoplyanik, A . (2010) The evolution of gas pricing: Europe \& CIS. Energy E conomist 347 September, 9-11.

LeB oeuf, Lamb, Greene \& M acRae, L.L.P. (2005) Russian law news: subsoil law amendments: two-key to one key, and more. 25 J anuary. A vailable at http://www.russianlaws.com/subsoil3.html.

McGowan, F. (2007) Can the European Union's Market Liberalism Ensure Security in a Time of "Economic Nationalism"?. J ournal of Contemporary European Research 4(2) 90-106.

Milov, V. (2005) Russian energy sector and its international implication. Institute of Energy Policy, M arch.

Riley, A. (2006) The Coming of the Russian Gas Deficit: Consequences and Solutions. Centre For European Policy Studies, Policy B riefing, 116.

Stern, J. (2006) Is Russia a Threat to Energy Supplies? Oxford Energy Forum, (66) 4-6.

Willems, A., Sul, J. and Benizri, Y . (2010) Unbundling as a Defence Mechanism Against Russia: Is the EU M issing the Point? 227-244 : In Talus, K., Fratini, P. (Eds) EU-Russia Energy Relations. OGEL collection, Euroconfidential. 